\title{
Oxytocin Modulates Neural Circuitry for Social Cognition and Fear in Humans
}

\author{
Peter Kirsch, ${ }^{1}$ Christine Esslinger, ${ }^{1}$ Qiang Chen, ${ }^{2,4}$ Daniela Mier, ${ }^{1}$ Stefanie Lis, ${ }^{1}$ Sarina Siddhanti, ${ }^{3,4}$ Harald Gruppe, ${ }^{1}$ \\ Venkata S. Mattay, ${ }^{2,4}$ Bernd Gallhofer, ${ }^{1}$ and Andreas Meyer-Lindenberg ${ }^{2,3,4}$ \\ ${ }^{1}$ Cognitive Neuroscience Group, Center for Psychiatry and Psychotherapy, Justus-Liebig University, D-35385 Giessen, Germany, and ${ }^{2}$ Neuroimaging Core \\ Facility, ${ }^{3}$ Unit for Systems Neuroscience in Psychiatry, and ${ }^{4}$ Clinical Brain Disorders Branch, Genes, Cognition, and Psychosis Program, National Institute \\ of Mental Health, National Institutes of Health, Department of Health and Human Services, Bethesda, Maryland 20892
}

In non-human mammals, the neuropeptide oxytocin is a key mediator of complex emotional and social behaviors, including attachment, social recognition, and aggression. Oxytocin reduces anxiety and impacts on fear conditioning and extinction. Recently, oxytocin administration in humans was shown to increase trust, suggesting involvement of the amygdala, a central component of the neurocircuitry of fear and social cognition that has been linked to trust and highly expresses oxytocin receptors in many mammals. However, no human data on the effects of this peptide on brain function were available. Here, we show that human amygdala function is strongly modulated by oxytocin. We used functional magnetic resonance imaging to image amygdala activation by fear-inducing visual stimuli in 15 healthy males after double-blind crossover intranasal application of placebo or oxytocin. Compared with placebo, oxytocin potently reduced activation of the amygdala and reduced coupling of the amygdala to brainstem regions implicated in autonomic and behavioral manifestations of fear. Our results indicate a neural mechanism for the effects of oxytocin in social cognition in the human brain and provide a methodology and rationale for exploring therapeutic strategies in disorders in which abnormal amygdala function has been implicated, such as social phobia or autism.

Key words: amygdala; social cognition; human; fMRI; oxytocin; fear

\section{Introduction}

Oxytocin is an evolutionarily highly preserved nonapeptide released from the paraventricular nucleus of the hypothalamus through the posterior pituitary (Buijs et al., 1983). Extensive work in diverse species including primates has established its importance as a central mediator of prosocial behavior (Insel and Fernald, 2004). In addition, oxytocin is released during stress (Jezova et al., 1995) and is an important modulator of anxiety and fear response (McCarthy et al., 1996).

Both complex social behavior (Adolphs, 2003) and basic emotional processing functions such as anxiety (LeDoux, 2000) and fear extinction critically depend on the amygdala. The lateral nucleus of the amygdala receives and integrates sensory and prefrontal/limbic inputs and excites, possibly indirectly, neurons in the central nucleus that evoke fear responses via their projections to brainstem regions including periaqueductal gray and reticular formation (LeDoux, 2000). In humans, fearful faces potently activate the amygdala (Whalen et al., 1998), and lesions of the amygdala impair recognition of fearful faces and lead to social

Received Sept. 12, 2005; revised 0ct. 30, 2005; accepted Nov. 6, 2005.

This work was supported in part by the Intramural Research Program of the National Institutes of HealthNational Institute of Mental Health. We thank Thomas Insel for helpful discussion of this manuscript and Joel Kleinman for support of this project.

Correspondence should be addressed to Dr. Andreas Meyer-Lindenberg, National Institutes of Health, 10-3C103, 9000 Rockville Pike, Bethesda, MD 20892-1365. E-mail: andreasml@nih.gov.

DOI:10.1523/JNEUROSCI.3984-05.2005

Copyright $\odot 2005$ Society for Neuroscience $\quad$ 0270-6474/05/2511489-05\$15.00/0 disinhibition (Adolphs et al., 2005) Decreased amygdala activation has been linked to genetic hypersociability (MeyerLindenberg et al., 2005) and increased instrumental aggression (Kiehl et al., 2001), whereas increased activation is observed in social avoidance and phobia (Stein et al., 2002). Consistent with the assumption that amygdala activation represents a danger signal in social interaction (Amaral, 2003), both conscious and implicit distrust of faces predicts amygdala activation (Winston et al., 2002), and trust is increased when the amygdala is damaged (Adolphs et al., 1998).

Animal models strongly suggest that the central role of oxytocin in mediating complex social behavior depends on the function of the amygdala: oxytocin acts on the amygdala to reduce fear (McCarthy et al., 1996) and modulate aggression (Bosch et al., 2005), and mouse knock-outs for oxytocin show a profound social recognition deficit despite normal olfactory and spatial learning abilities that can be fully restored by injection of oxytocin in the medial amygdala (Ferguson et al., 2001). In addition, recent data show that oxytocin acts on the central amygdala to inhibit excitatory flow from the amygdala to brainstem sites mediating fear response (Huber et al., 2005). Based on these data, we hypothesized that oxytocin would reduce amygdala activation in humans and modulate its participation in functional networks related to fear processing.

We used two visual matching tasks requiring perceptual processing of threatening visual stimuli of different social valence that reliably engage the amygdala and have been shown to be 
sensitive to genetic mechanisms of abnormal social behavior (Meyer-Lindenberg et al., 2005) and risk for anxiety (Pezawas et al., 2005). In a double-blind experiment, 15 male participants applied oxytocin or placebo intranasally, a modality shown to reliably deliver neuropeptides to the brain (Born et al., 2002).

\section{Materials and Methods}

Subjects. Participants (age, $26.7 \pm 3.0$ years; 13 right-handed) were required to be normotensive males between 18 and 40 years of age with normal magnetic resonance imaging (MRI) and without a history or signs of neurological, psychiatric, or serious somatic illness. All participants participated in the study after written informed consent and were reimbursed for their time. The project was reviewed and approved by the Ethics Board of the University of Giessen and the National Institutes of Health Office of Human Subjects Research. Of the initially recruited participants, one subject was excluded after pathological findings were obtained on screening MRI and replaced by another.

Experimental paradigm. Functional MRI (fMRI) experiments were scheduled with an interval of 1-2 weeks as part of a larger study on neuropeptide effects. Subjects abstained from alcohol and nicotine for $12 \mathrm{~h}$ before the experiment. Drug distribution was double-blind and counterbalanced for order. Thirty minutes before the start of the fMRI session, subjects intranasally self-applied five puffs of oxytocin (27 IU total) or placebo (containing only carrier substance) under experimenter supervision. The oxytocin dose was comparable to that used previously in human studies of behavior (Kosfeld et al., 2005). On informal questioning during debriefing, subjects were unable to discriminate placebo from oxytocin.

Before drug administration and after the scan, subjects completed the following scales: the State-Trait Anger Expression Inventory (STAXI; trait component only after scanning) (Spielberger, 1988), the SelfAssessment Manikin (SAM) (Bradley and Lang, 1994), and the Multidimensional Comfort Questionnaire (MDBF) (Steyer et al., 1994). Statesensitive instruments (STAXI state component, SAM, and MBDF) were administered before and after drug administration to investigate an acute effect of oxytocin on the measured scales.

After an initial positioning and anatomical scan, fMRI imaging commenced at $50 \mathrm{~min}$ after drug application. The fMRI paradigm was similar to experiments described previously (Hariri et al., 2002; MeyerLindenberg et al., 2005; Pezawas et al., 2005). In the first task, one of two simultaneously presented angry or afraid faces was matched with an identical target face of identical expression. In the other task, participants matched one of two simultaneously presented fearful/threatening scenes with an identical target scene (Hariri et al., 2002). As a control, participants matched simple shapes (circles or ellipses). The scenes were selected from a larger set to be devoid of social interaction or facial displays and should therefore represent nonsocially relevant threatening stimuli, whereas threatening and angry faces are socially relevant stimuli, an assumption supported by previous research with this paradigm (MeyerLindenberg et al., 2005). It consisted of 18 experimental blocks of $32 \mathrm{~s}$ : four each for matching facial expressions and scenes, interleaved with 10 control blocks, for a total scan length of $9.2 \mathrm{~min}$. Image order was counterbalanced across subjects and visits. Each block began with a 2 s instruction (in German): "Match Faces," "Match Pictures," or "Match Forms," followed by six matching panels displayed for $5 \mathrm{~s}$ each, randomized for all conditions. Subjects responded by pressing one of two buttons with their right hand.

Functional imaging. Blood oxygenation level-dependent (BOLD) fMRI was performed on a General Electric (Milwaukee, WI) Signa 1.5T using gradient echo planar imaging (30 axial slices; $5 \mathrm{~mm}$ thickness; repetition time, $3000 \mathrm{~ms}$; echo time, $50 \mathrm{~ms}$; field of view, $22 \mathrm{~cm}$; matrix, $64 \times 64$ ). Images were processed as described previously (Pezawas et al., 2005) using SPM2 (http://www.fil.ion.ucl.ac.uk/spm/software/download.html). Briefly, images were realigned, spatially normalized into a standard stereotactic space [Montreal Neurological Institute (MNI) template], resliced to $3 \mathrm{~mm}$ isotropic voxels, and smoothed with a $10 \mathrm{~mm}$ full-width at half-maximum Gaussian filter. A statistical image for the contrast of fearful versus control stimuli was obtained for each stimulus type and subject and analyzed in a second-level random-effects model (ANOVA) for significant differences in amygdala activation between the oxytocin and the placebo condition. The threshold for significance was set at $p<0.05$, corrected for multiple comparisons in the amygdala region of interest (ROI). As described previously (Pezawas et al., 2005), this ROI was defined by a combined functional and anatomical criterion by selecting all voxels in the anatomical amygdala region [according to the Wake Forest University (WFU) PickAtlas software; http://www. fmri.wfubmc.edu/download.htm] that were significantly $(p<0.05)$ activated during the matching compared with the control task across drug conditions.

Functional connectivity. "Functional connectivity" was investigated as described previously (Pezawas et al., 2005). This measure examines the covariation across the brain with the activation in a region (volume) of interest. As a seed region, we used the same amygdala mask as for functional activation. After mean and drift correction of the time series, median activity within this ROI was calculated for each scan. These values were then correlated across the brain with all voxel time series, resulting in a map that contained, in each voxel, the correlation coefficient of the time series in this voxel with that of the reference region. These maps, one per subject, were then analyzed in a random-effects model in SPM as described above for activation. For assessment of corrected significance, again set at $p<0.05$, corrected for multiple comparisons, a brainstem ROI was derived from the WFU PickAtlas software by restricting the entire brainstem mask to the region of the midbrain (between $z$ coordinates -2 and -20$)$. In contrast to the stringent threshold used for statistical significance testing, all activation figures were thresholded leniently for display ( $p<0.01$, uncorrected, unmasked) to give an accurate impression of residual activation and connectivity remaining under the oxytocin condition.

\section{Results}

In behavioral testing, the neuropeptide had no effect on task performance, anger scales, dominance, or arousal (Table 1). As expected, the subject felt less awake after the scan session, but this effect did not interact with drug condition. A nominally significant ( $p<0.04$, uncorrected) higher emotional valence before the scan session during the placebo condition in the SAM scale is likely to be a type II error, because the corresponding scale in the MDBF showed no such effect $(p=0.56)$ and would not survive multiple comparison correction (Table 1 ).

Confirming previous results (Hariri et al., 2002), the fMRI experiment showed strong activation of the amygdala to both classes of stimuli during the placebo condition (Fig. 1), predominantly on the left. Compared with placebo, oxytocin significantly depressed amygdala activation (Fig. $1 \mathrm{~B}$, Table 2) (maximum effect at the left amygdala; coordinates: $-24,3,-24 ; T=$ $2.59 ; p<0.006)$. Testing the stimulus types separately showed that this effect was more pronounced for faces (socially relevant stimuli) than scenes (Table 2, Fig. 1).

In addition to the effects on amygdala activation, it was demonstrated recently in rats that oxytocin may also disrupt the common output from the amygdala to the brainstem effector sites of the autonomic nervous system by action on a distinct neuronal population in the central nucleus (Huber et al., 2005). Although the resolution of fMRI is not currently sufficient to resolve intraamygdalar processing, we investigated whether functional coupling of the human amygdala to the brainstem was influenced by oxytocin. To this end, we studied the functional connectivity of the amygdala, a measure of correlated activity in BOLD over time that is widely used as a simple and robust characterization of functionally relevant neural interaction (Friston et al., 1993). We found that the amygdala was functionally connected to the upper brainstem during the placebo condition and that this connectivity was significantly reduced under oxytocin, in excellent agreement with the data acquired in animal models (Fig. 2, Table 2). 
Table 1. Behavioral data

\begin{tabular}{|c|c|c|c|c|c|}
\hline & Placebo before scan & Placebo after/during scan & 0xytocin before scan & 0xytocin after/during scan & $t$ test (after/during scanning) \\
\hline \multicolumn{6}{|l|}{ Faces task } \\
\hline Accuracy (\%) & & $98.8 \%$ (13.3) & & $99.1 \%(1.7)$ & $p=0.77$ \\
\hline Reaction time (ms) & & $1094(205)$ & & 1105 (194) & $p=0.91$ \\
\hline \multicolumn{6}{|l|}{ Scene task } \\
\hline Accuracy (\%) & & $99.7 \%(1.0)$ & & $100 \%(0.0)$ & $p=1.00$ \\
\hline Reaction time (ms) & & 898 (171) & & $899(140)$ & $p=0.99$ \\
\hline \multicolumn{6}{|l|}{ STAXI ${ }^{a}$} \\
\hline State & $10.1(0.4)$ & $10.2(0.8)$ & $10.6(1.8)$ & $10.3(0.8)$ & $p=0.33$ \\
\hline Trait & & $17.7(3.8)$ & & $18.3(3.6)$ & $p=0.23$ \\
\hline \multicolumn{6}{|l|}{$\mathrm{MDBF}^{b}$} \\
\hline Arousal & $31.3(6.3)$ & $34.1(6.0)$ & $33.9(4.8)$ & $34.1(6.8)$ & $p=0.96$ \\
\hline Wakefulness & $30.1(9.2)$ & $24.1(8.9)$ & $32.5(6.6)$ & $26.2(7.4)$ & $p=0.28$ \\
\hline Emotional valence ${ }^{c}$ & $34.2(4.8)$ & $35.0(4.5)$ & $35.5(3.7)$ & $35.6(5.4)$ & $p=0.56$ \\
\hline \multicolumn{6}{|l|}{$\mathrm{SAM}^{d}$} \\
\hline Arousal & $3.7(0.98)$ & $4.3(0.70)$ & $3.8(0.68)$ & $4.1(0.96)$ & $p=0.42$ \\
\hline Dominance & $3.5(0.64)$ & $3.6(0.63)$ & $3.8(0.56)$ & $3.6(0.83)$ & $p=1.00$ \\
\hline Emotional valence $^{c}$ & $1.9(0.92)$ & $1.5(0.64)$ & $1.6(0.51)$ & $1.7(0.80)$ & $p=0.04$ \\
\hline
\end{tabular}

A nominally significant reduction in valence in the SAM was not seen in the MDBF. The mean (SD) is shown. ANOVA of drug-by-time of application interactions did not identify any significant effects. Wakefulness (MDBF) was significantly reduced after the scan.

${ }^{a}$ Data are from Spielberger (1988).

${ }^{b}$ One subject failed to fill out a questionnaire for the MDBF (Steyer et al., 1994).

'Higher equals more positive.

${ }^{d}$ Data are from Bradley and Lang (1994).
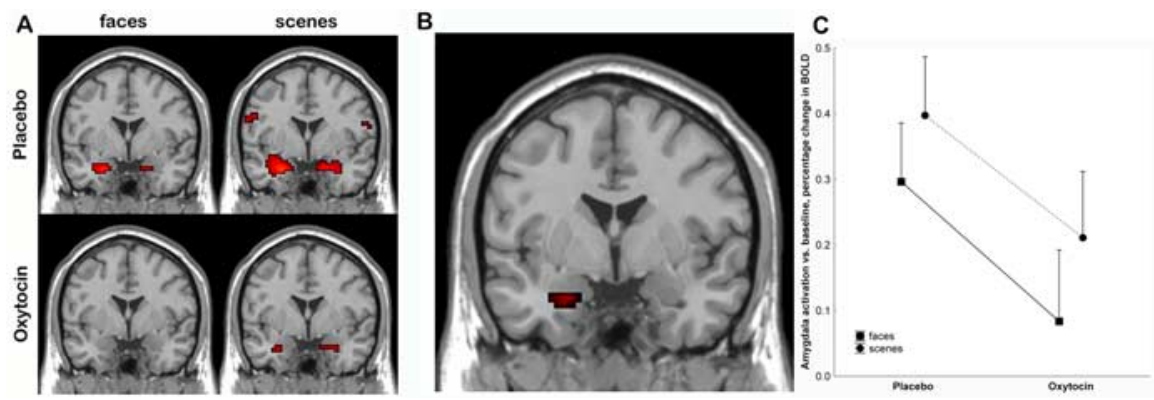

Figure 1. Oxytocin effects on amygdala activation. $A$, Rendering on normal coronal MRI at the level of the anterior commissure (in neurological orientation: the brain left is on the viewer's left). The response to face stimuli is on the left, and the response to scene stimuli is on the right. Top, Placebo; bottom, oxytocin. See Table 2 for statistical information. $\boldsymbol{B}$, Significantly higher activation under placebo than oxytocin (main effect of drug condition). See Table 2 for statistical information. $C$, Plot of BOLD in the amygdala ROI (ANOVA; significant main effect of drug condition: $F_{(1,56)}=4.2, p=0.045$; main effect of task and drug-by-task interaction were not significant).

Table 2. Significant differences in amygdala activation and connectivity

\begin{tabular}{lll}
\hline Area & Talairach coordinates & Tvalue \\
\hline $\begin{array}{l}\text { Activation } \\
\quad \text { Placebo > oxytocin }\end{array}$ & & \\
$\quad$ Left amygdala (main effect) & $-24,3,-24$ & 2.59 \\
$\quad$ Left amygdala (faces task) & $-24,3,-24$ & 2.12 \\
$\quad$ Left amygdala (scenes task) & $-21,0,-24$ & $1.88^{*}$ \\
$\begin{array}{l}\text { Connectivity } \\
\text { Placebo }>\text { oxytocin }\end{array}$ & & \\
$\quad$ Midbrain & & \\
$\quad$ Midbrain & $9,-27,-15$ & 3.50 \\
\end{tabular}

All reported voxels are significant at $p<0.05$, corrected for multiple comparisons within the amygdala ROI (activation) or brainstem ROI (connectivity), except for ${ }^{*} p=0.077$, corrected. Coordinates are in millimeters relative to the anterior commissure in the space defined by the MNI template.

\section{Discussion}

Using a well validated paradigm to robustly activate the amygdala with two classes of fearful visual stimuli, we found a pronounced reduction in activation and amygdala-midbrain connectivity in healthy male subjects receiving oxytocin in a double-blind study.
Because amygdala activation is critical for signaling of fear (Amaral, 2003; Adolphs et al., 2005), our data fit well with the reported anxiolytic effects of oxytocin (McCarthy et al., 1996; Heinrichs et al., 2003) and its mediation by neuropeptide effects on the amygdala in animals (McCarthy et al., 1996; Bosch et al., 2005). It should be noted, however, that the only human postmortem study on oxytocin receptor distribution, using autoradiography, did not find evidence for specific binding in the amygdala (Loup et al., 1991), unlike other mammalian and primate species studied (Winslow and Insel, 2004). Although this could argue for an extra-amygdalar origin of the effects observed here, the results of the present in vivo study suggest that this issue should be revisited in vitro. In excellent agreement with preclinical data suggesting a selective effect of oxytocin on amygdalar output (Huber et al., 2005), we found an additional impact of the neuropeptide on functional connectivity between amygdala and brainstem effector sites of the fear response. This effect was located on the level of the midbrain and encompassed both the region of the periaqueductal gray and of the reticular formation (Fig. 2), which are prominent among the brainstem areas to which the central nucleus of the amygdala projects (LeDoux, 2000) and which mediate fear behavior and arousal (LeDoux et al., 1988). This indicates that the effect of oxytocin on anxiety in humans may be attributable to a combined effect on both amygdala activation and coupling to regions mediating fear response. In agreement with our findings, autonomic response to aversive pictures has been reported previously to be reduced under oxytocin (Pitman et al., 1993), compatible with the effect on amygdala activation and connectivity seen here. Because increased amygdala activation has been associated with social fear in social phobia (Stein et al., 2002), genetic risk for anxiety and depression (Pezawas et al., 2005), and possibly with social fear in autism 

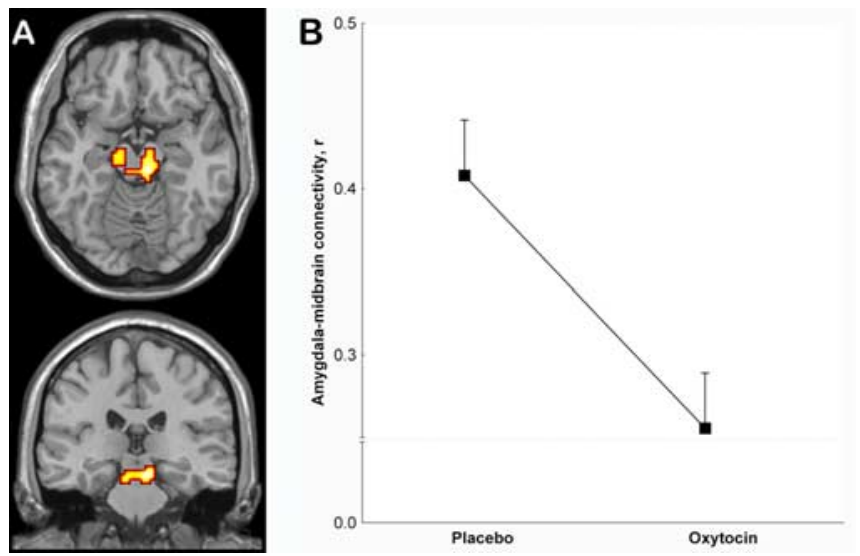

Figure 2. Significant decrease in coupling of the amygdala to the midbrain under oxytocin. $\boldsymbol{A}$, Maps of significant difference in connectivity from the amygdala to the midbrain, in neurological orientation. See Table 2 for statistical information. $\boldsymbol{B}$, Plot of oxytocin effect on correlation with the amygdala (Pearson's $r$ ) at the midbrain location showing maximum linkage to the amygdala during the placebo condition (coordinates: $-6,-24,-15)$, highly significant decrease under oxytocin ( $p<0.004 ; t$ test).

assessed during faces processing (Dalton et al., 2005), this dual mode of action of oxytocin in humans suggests a potential powerful treatment approach toward socially relevant fear.

Because previous studies linked increased amygdala activation to both overt and implicit lack of trust in displayed faces (Winston et al., 2002), our finding of reduced amygdala activation under oxytocin agrees with our hypothesis about a contribution of reduced amygdalar danger signaling to increased trust. It is of interest to note that oxytocin administration did not affect self-report scales of psychological state (Table 1). This agrees with the observations of Kosfeld et al. (2005), who also did not find an effect of oxytocin on measured calmness and mood and showed that on the level of behavior, actual social interaction was necessary to bring out the oxytocin effect. Our data indicate that fMRI is sensitive enough to show changes in neural processing that lead up to this bias in behavior observed during social interaction.

In addition to trust, reduced amygdala activation to fearful faces has been linked to increased sociability and decreased social fear in humans (Adolphs et al., 2005; Meyer-Lindenberg et al., 2005), and the neuropeptide action observed here may therefore contribute to the prosocial effects of oxytocin (Insel and Fernald, 2004). In this context, we note that the reduction in amygdala activation was more significant for socially relevant stimuli (faces) than for the socially less relevant scenes; differential impairment of amygdala signaling related to the social relevance of the stimuli is in agreement with emerging primate lesion (Prather et al., 2001) and human (Meyer-Lindenberg et al., 2005) data indicating that social and nonsocial fear may depend on dissociable neural systems. However, inspection of activation magnitude by condition (Fig. $1 B$ ) does not support such a distinction, because the reduction in activation under oxytocin was similar for both stimulus groups.

In rats, oxytocin has been shown to increase aggression toward intruders (Bosch et al., 2005) but inhibits aggression against pups. Conversely, oxytocin knock-out mice show increased unprovoked aggression and are infanticidal (Ragnauth et al., 2005). This indicates that the contribution of this neuropeptide to aggressive behavior is complex and depends on the specifics of the social interaction. This is further confirmed by findings in nonhuman primates, in which oxytocin had opposing effects on aggression in males depending on social dominance status (Win- slow and Insel, 1991). In humans, an important distinction has been drawn between reactive and instrumental aggression (Blair, 2003), with only the latter being associated with impaired amygdala structure and function (Kiehl et al., 2001), especially in males. Our data would therefore predict a reduction in reactive aggression under oxytocin in humans, but this requires further study under social contexts. In this regard, it is again noteworthy that behavioral scales of anger showed no effect of oxytocin in this experiment. This finding, which agrees with previous observations (Kosfeld et al., 2005), again indicates that the neural effect of the neuropeptide identified using sensitive fMRI methodology biases behavior in the social context but not when subjects rate themselves in isolation.

In this study, we opted for the use of a low-level baseline (matching forms) because this has been shown to be necessary for reliable and reproducible amygdala activation (Johnstone et al., 2005), essential for the double-blind design used here. Future work could now explore differential activation to other socially relevant stimuli, such as neutral or happy faces, to further explore neuropeptide effects on social cognition. In addition to the study of amygdalar output pathways presented here, it will also be informative to investigate the extended neural circuits of which the amygdala is a part. For example, oxytocin modulates fear extinction (Ibragimov, 1990), which depends on medial prefrontalamygdalar interactions (Phelps et al., 2004) that were shown previously to predict trait anxiety using the task applied here (Pezawas et al., 2005). Given the pronounced gender differences in neuropeptide action in several species (Winslow and Insel, 2004), the study of females will also be of interest. Further characterization of amygdala interactions will also be important for clinical applications; for example, in autism, in which plasma oxytocin is reduced (Green et al., 2001), abnormal amygdala activation may depend on face processing (Dalton et al., 2005) regions, and genetically determined amygdala dysregulation by the prefrontal cortex (Meyer-Lindenberg et al., 2005) has been demonstrated.

In conclusion, our data reveal a pronounced impact of oxytocin on amygdala reactivity and brainstem interactions in humans, extending a large body of work on neuropeptide regulation of complex behavior to this species by establishing an effect of oxytocin on a key component of affective and social processing. We hope that this work will contribute to the development of therapeutic interventions with oxytocin or synthetic agonists in diseases in which amygdala dysfunction has been implicated, including anxiety disorders, depression, and autism.

\section{References}

Adolphs R (2003) Cognitive neuroscience of human social behaviour. Nat Rev Neurosci 4:165-178.

Adolphs R, Tranel D, Damasio AR (1998) The human amygdala in social judgment. Nature 393:470-474.

Adolphs R, Gosselin F, Buchanan TW, Tranel D, Schyns P, Damasio AR (2005) A mechanism for impaired fear recognition after amygdala damage. Nature 433:68-72.

Amaral DG (2003) The amygdala, social behavior, and danger detection. Ann NY Acad Sci 1000:337-347.

Blair RJ (2003) Neurobiological basis of psychopathy. Br J Psychiatry 182:5-7.

Born J, Lange T, Kern W, McGregor GP, Bickel U, Fehm HL (2002) Sniffing neuropeptides: a transnasal approach to the human brain. Nat Neurosci 5:514-516.

Bosch OJ, Meddle SL, Beiderbeck DI, Douglas AJ, Neumann ID (2005) Brain oxytocin correlates with maternal aggression: link to anxiety. J Neurosci 25:6807-6815.

Bradley MM, Lang PJ (1994) Measuring emotion: the Self-Assessment 
Manikin and the Semantic Differential. J Behav Ther Exp Psychiatry 25:49-59.

Buijs RM, De Vries GJ, Van Leeuwen FW, Swaab DF (1983) Vasopressin and oxytocin: distribution and putative functions in the brain. Prog Brain Res 60:115-122.

Dalton KM, Nacewicz BM, Johnstone T, Schaefer HS, Gernsbacher MA, Goldsmith HH, Alexander AL, Davidson RJ (2005) Gaze fixation and the neural circuitry of face processing in autism. Nat Neurosci 8:519-526.

Ferguson JN, Aldag JM, Insel TR, Young LJ (2001) Oxytocin in the medial amygdala is essential for social recognition in the mouse. J Neurosci 21:8278-8285.

Friston KJ, Frith CD, Liddle PF, Frackowiak RS (1993) Functional connectivity: the principal-component analysis of large (PET) data sets. J Cereb Blood Flow Metab 13:5-14.

Green L, Fein D, Modahl C, Feinstein C, Waterhouse L, Morris M (2001) Oxytocin and autistic disorder: alterations in peptide forms. Biol Psychiatry 50:609-613.

Hariri AR, Tessitore A, Mattay VS, Fera F, Weinberger DR (2002) The amygdala response to emotional stimuli: a comparison of faces and scenes. NeuroImage 17:317-323.

Heinrichs M, Baumgartner T, Kirschbaum C, Ehlert U (2003) Social support and oxytocin interact to suppress cortisol and subjective responses to psychosocial stress. Biol Psychiatry 54:1389-1398.

Huber D, Veinante P, Stoop R (2005) Vasopressin and oxytocin excite distinct neuronal populations in the central amygdala. Science 308:245-248.

Ibragimov R (1990) Influence of neurohypophyseal peptides on the formation of active avoidance conditioned reflex behavior. Neurosci Behav Physiol 20:189-193.

Insel TR, Fernald RD (2004) How the brain processes social information: searching for the social brain. Annu Rev Neurosci 27:697-722.

Jezova D, Skultetyova I, Tokarev DI, Bakos P, Vigas M (1995) Vasopressin and oxytocin in stress. Ann NY Acad Sci 771:192-203.

Johnstone T, Somerville LH, Alexander AL, Oakes TR, Davidson RJ, Kalin $\mathrm{NH}$, Whalen PJ (2005) Stability of amygdala BOLD response to fearful faces over multiple scan sessions. NeuroImage 25:1112-1123.

Kiehl KA, Smith AM, Hare RD, Mendrek A, Forster BB, Brink J, Liddle PF (2001) Limbic abnormalities in affective processing by criminal psychopaths as revealed by functional magnetic resonance imaging. Biol Psychiatry 50:677-684.

Kosfeld M, Heinrichs M, Zak PJ, Fischbacher U, Fehr E (2005) Oxytocin increases trust in humans. Nature 435:673-676.

LeDoux JE (2000) Emotion circuits in the brain. Annu Rev Neurosci 23:155-184.

LeDoux JE, Iwata J, Cicchetti P, Reis DJ (1988) Different projections of the central amygdaloid nucleus mediate autonomic and behavioral correlates of conditioned fear. J Neurosci 8:2517-2529.

Loup F, Tribollet E, Dubois-Dauphin M, Dreifuss JJ (1991) Localization of high-affinity binding sites for oxytocin and vasopressin in the human brain. An autoradiographic study. Brain Res 555:220-232.

McCarthy MM, McDonald CH, Brooks PJ, Goldman D (1996) An anxiolytic action of oxytocin is enhanced by estrogen in the mouse. Physiol Behav 60:1209-1215.

Meyer-Lindenberg A, Hariri AR, Munoz KE, Mervis CB, Mattay VS, Morris CA, Berman KF (2005) Neural correlates of genetically abnormal social cognition in Williams syndrome. Nat Neurosci 8:991-993.

Pezawas L, Meyer-Lindenberg A, Drabant EM, Verchinski BA, Munoz KE, Kolachana BS, Egan MF, Mattay VS, Hariri AR, Weinberger DR (2005) 5-HTTLPR polymorphism impacts human cingulate-amygdala interactions: a genetic susceptibility mechanism for depression. Nat Neurosci 8:828-834.

Phelps EA, Delgado MR, Nearing KI, LeDoux JE (2004) Extinction learning in humans: role of the amygdala and vmPFC. Neuron 43:897-905.

Pitman RK, Orr SP, Lasko NB (1993) Effects of intranasal vasopressin and oxytocin on physiologic responding during personal combat imagery in Vietnam veterans with posttraumatic stress disorder. Psychiatry Res 48:107-117.

Prather MD, Lavenex P, Mauldin-Jourdain ML, Mason WA, Capitanio JP, Mendoza SP, Amaral DG (2001) Increased social fear and decreased fear of objects in monkeys with neonatal amygdala lesions. Neuroscience 106:653-658

Ragnauth AK, Devidze N, Moy V, Finley K, Goodwillie A, Kow LM, Muglia LJ, Pfaff DW (2005) Female oxytocin gene-knockout mice, in a seminatural environment, display exaggerated aggressive behavior. Genes Brain Behav 4:229-239.

Spielberger CD (1988) State Trait Anger Expression Inventory (STAXI), research edition. Odessa, FL: Psychological Assessment Resources.

Stein MB, Goldin PR, Sareen J, Zorrilla LT, Brown GG (2002) Increased amygdala activation to angry and contemptuous faces in generalized social phobia. Arch Gen Psychiatry 59:1027-1034.

Steyer R, Schwenkmezger P, Notz P, Eid M (1994) Testtheoretische Analysen des mehrdimensionalen Befindlichkeitsfragebogens (MDBF). Diagnostica 40:320-328.

Whalen PJ, Rauch SL, Etcoff NL, McInerney SC, Lee MB, Jenike MA (1998) Masked presentations of emotional facial expressions modulate amygdala activity without explicit knowledge. J Neurosci 18:411-418.

Winslow JT, Insel TR (1991) Social status in pairs of male squirrel monkeys determines the behavioral response to central oxytocin administration. J Neurosci 11:2032-2038.

Winslow JT, Insel TR (2004) Neuroendocrine basis of social recognition. Curr Opin Neurobiol 14:248-253.

Winston JS, Strange BA, O’Doherty J, Dolan RJ (2002) Automatic and intentional brain responses during evaluation of trustworthiness of faces. Nat Neurosci 5:277-283. 